\title{
分子篮限域孔道中吡啶的吸附结构和能量
}

\author{
韩 冰 ${ }^{1,2}$ 褚月英 ${ }^{1,2}$ 郑安民 ${ }^{1, *}$ 邓 风1,* \\ ('中国科学院武汉物理与数学研究所, 波谱与原子分子物理国家重点实验室, 武汉核磁共振中心, 武汉 430071; \\ ${ }^{2}$ 中国科学院研究生院, 北京 100049)
}

\begin{abstract}
摘要: 以吸附于 ZSM- 5 孔道中的吡啶分子为例, 利用量子化学理论方法考察了计算模型和密度泛函方法的选 择对吡啶吸附结构和吸附能的影响, 从而为准确计算分子篎限域孔道中客体分子吸附态结构和能量参数提供 了依据. 计算结果表明, 吡啶吸附能随着所选用的分子篮的计算模型(从 $8 \mathrm{~T}$ 到 128T)增大而增大, 当选用的孔道 结构能够将整个分子篮的孔道结构完全包括进来的时候(72T)达到收玫. 与常规的密度泛函方法(B3LYP 和 M06-2X)相比较, 考虑到色散作用校正的 B97D泛函方法能够很好地处理分子笁体系中主客体间的长程相互作 用和弱相互作用, 计算得到的能量数据与实验结果符合得很好.
\end{abstract}

关键词: 量子化学计算; 分子篮; 限域效应; 吸附能 中图分类号: 0641

\section{Adsorption Structure and Energy of Pyridine Confined inside Zeolite Pores}

\author{
HAN Bing ${ }^{1,2}$ CHU Yue-Ying ${ }^{1,2} \quad$ ZHENG An-Min ${ }^{1, *} \quad$ DENG Feng ${ }^{1, *}$ \\ ( ${ }^{1}$ Wuhan Center for Magnetic Resonance, State Key Laboratory of Magnetic Resonance and Atomic and Molecular Physics, \\ Wuhan Institute of Physics and Mathematics, Chinese Academy of Sciences, Wuhan 430071, P. R. China; \\ ${ }^{2}$ Graduate University of Chinese Academy of Sciences, Beijing 100049, P. R. China)
}

\begin{abstract}
The performance of different exchange-correlation functionals for the description of the interaction of pyridine with different cluster models of ZSM-5 zeolite has been assessed. Theoretical calculations show that upon increasing the cluster model from $8 \mathrm{~T}$ to $128 \mathrm{~T}$, the adsorption energy of pyridine in ZSM-5 zeolite increases gradually and reaches convergence by the $72 \mathrm{~T}$ cluster model. On the basis of the $72 \mathrm{~T}$ cluster model, the pyridine adsorption energy calculated with different functionals is further examined. Compared to the conventional functionals (B3LYP and M06-2X), the B97D functional which takes into account the dispersion correction provides calculated results that agree well with experimental data. The present results indicate that the B97D functional is suitable for studying long-range interactions in weakly interacting systems.
\end{abstract}

Key Words: Quantum chemical calculation; Zeolite; Confinement effect; Adsorption energy

1 引言

分子篮催化剂因为环境友好而在石油化工中
被广泛应用. 作为固体酸催化剂, 它具有不腐蚀设 备、容易与液相反应体系分离、环境污染小、选择性

\footnotetext{
Received: October 9, 2011; Revised: November 17, 2011; Published on Web: November 23, 2011.

"Corresponding authors. ZHENG An-Min, Email: zhenganm@wipm.ac.cn; Tel: +86-27-87197359. DENG Feng, Email: dengf@wipm.ac.cn; Tel: +86-27-87198820.

The project was supported by the National Natural Science Foundation of China (21073228, 20933009, 20921004) and National Key Basic Research Program of China (973) (2009CB918600).

国家自然科学基金(21073228, 20933009, 20921004)及国家重点基础研究发展规划项目(973) (2009CB918600)资助
}

(C) Editorial office of Acta Physico-Chimica Sinica 
高等特点. ${ }^{1}$ 由于分子篎具有独特的孔道特征、较好 的热稳定性和优越的离子交换能力, ${ }^{2}$ 因此在裂解、 质子化、异构化和聚合等反应中有广泛的应用. ${ }^{3}$ 分 子篮的酸性特征直接与催化活性相关联, 研究分子 篮的表面酸性对于深入理解其催化活性及催化机 理具有重要意义. 45 吡啶作为一种碱性探针分子被 广泛地用来表征固体酸催化剂的酸强度, 例如在程 序升温吸脱附(TPD) 方法中, 通过比较吡啶脱附温 度的高低来确定酸强度的大小; 而在核磁共振 (NMR)探针分子方法中, 通过比较吡啶吸附前后的 ${ }^{1} \mathrm{H}$ 化学位移变化来定量测量酸强度. ${ }^{6}$ 通过吡啶的 TPD 和 NMR 测定方法, 人们可以很直观地比较催 化剂的酸强度大小, 而这些谱学参数与吡啶在酸位 表面的吸附构型和吸附热参数是密切相关的. ${ }^{7}$ 由此 看来, 研究吡啶与分子笁酸位的主客体相互作用的 结构和能量(吸附热)参数对于在原子分子水平下解 释实验谱学参数显得特别重要, 然而这些重要信息 并不能通过实验方法来直接获取.

理论计算的方法作为实验方法有力的补充, 已 经被广泛用来分析固体催化剂活性中心的结构和 催化反应机理. 彭少逸等 ${ }^{8}$ 研究了 $\mathrm{B} 、 \mathrm{Al} 、 \mathrm{Ga}$ 等同晶 取代进入丝光沸石骨架后可能存在的位置, 考察了 杂原子进入骨架对丝光沸石 Brönsted 酸性的影响, 孙淮等 ${ }^{9}$ 研究了磷在 P-ZSM-5 沸石中存在的形态, Yang 等 ${ }^{10}$ 研究了 La 离子在分子篮骨架中的存在形 态和 Lewis 酸性特征, Zhou 等 ${ }^{11}$ 研究了钼掺杂的 ZSM-5 分子篮上甲烷脱氢芳构化反应路径. 在众多 的量子化学理论方法中, 密度泛函理论(DFT) 是目 前应用最广泛的理论计算方法之一, 该方法不需要 很大的计算资源却得到不错的精确度, 因此被广泛 应用到有机体系热力学和动力学的预测. ${ }^{12,13}$

分子篮催化剂由于具有纳米量级的三维孔道 结构而在石油化工领域有着广泛的应用, 其独特产 物的选择性源于客体分子(反应物、过渡态、产物或 反应中间体)与分子篎限域孔道中的吸附或排斥作 用. 孔道限域效应主要表现为吸附分子与分子篮孔 道之间的长程主客体相互作用 (如范德华作用). 众 所周知, 经典的 DFT泛函并不能用来精确描述范德 华作用力以及氢键这类弱相互作用, 理论预测的吸 附能往往较实验值偏低. ${ }^{14}$ 因此, 建立新的理论方法 来准确描述氢键和范德华作用力是当前理论研究 的热点, 其中 Becke 小组 ${ }^{15}$ 在 1998 年开发出混合梯 度近似泛函如 B97 系列, 这种泛函对于研究短程作
用为主的体系有较高的计算精度. 后来, Truhlar等 ${ }^{16}$ 于 2006年研发了M06系列泛函(M06、M06-L、M06$2 \mathrm{X}$ ), 研究结果表明这一系列泛函能够更好地描述 非键相互作用. 这是因为色散力作为范德华作用力 的主要来源, 在研究分子间的相互作用时非常重要 而不能被忽视. 可以设想, 如果在理论计算中能够 将色散相互作用考虑进来, 那么计算得到的吸附结 构和吸附能参数将更接近于实验值. Grimme ${ }^{17}$ 于 2004 年提出了 DFT-D算法, 利用半经验的 $-C_{6} / R^{6}$ 形 式的色散项来表示范德华作用力. 在 DFT-D算法 中, 系统的总能量 $\left(E_{\mathrm{DFF}-\mathrm{D}}\right)$ 表示为 DFT 计算得到的能 量 $\left(E_{\mathrm{DFT}}\right)$ 和长程相互作用的色散能贡献 $\left(E_{\mathrm{disp}}\right)$ 之和, 即: $E_{\mathrm{DFT}-\mathrm{D}}=E_{\mathrm{DFT}}+E_{\text {disp. }}$. 其中长程相互作用的色散能贡 献 $E_{\text {disp }}$ 对于短程作用为零, 则回归为 DFT泛函的原 始形式, 不影响泛函本身在短程相关效应描述较好 的原有优点. DFT-D解决了 DFT 泛函不能很好地描 述弱相互作用及长程作用中的范德华作用的不足, 因而引起了人们越来越多的兴趣. 基于 B 3 LYP 而改 进的 B3LYP-D 算法, 是利用在 B 3 LYP 方法优化的 基础上, 在最后加上色散校正项而得到最终的单点 能参数; 同样 B97-D 方法与 B3LYP-D 方法类似, 在 单点能部分加上色散作用的校正. 两种方法的优势 还在于占用的计算资源不大, 计算精度也比较高. 而 B97D 方法则与 B97-D 方法有所不同, 它是在结 构优化的每一步都将色散相互作用考虑进来. ${ }^{18}$

准确描述有机物在分子篮孔道中的吸附行为 的理论研究对于解释实验现象、揭示反应机理是非 常重要的. 目前B3LYP、M06-2X、B97D等方法广泛 用于研究分子篎的酸性特征, 预测分子篮催化机理 和过渡态特征. ${ }^{19-22}$ 然而, 哪种泛函方法得到的结构 参数和吸附能与实验更为接近, 文献一直没有相关 的报导. 所以有必要通过系统考察B3LYP、B971、 M06-2X 以及考虑色散校正作用的 B3LYP-D、 B971-D 和 B97D 等 6 种理论方法的计算结果, 与实 验结果对比来确定能够准确预测有机物种在分子 篎孔道中吸附结构和能量的泛函方法. 本文拟从分 子篮计算模型和理论方法的选择两个方面入手, 探 索出能够准确预测有机分子在分子篮孔道中结构 参数和吸附能量的准确方法.

\section{2 计算方法}

\section{1 模型的选取}

为了考察分子篮孔道结构对 Brönsted 酸位和吡 
啶吸附的影响, 分别选取 8T、30T、46T、60T、72T 和 $128 \mathrm{~T}$ 团簇模型来表示分子篎 ZSM-5 的孔道结构(见 图 1). 需要指出的是, $8 \mathrm{~T}$ 模型仅将 Brönsted 酸活性 中心的局域结构考虑进来, 随着模型的逐渐增大, 分子篮完整的孔道结构将被重现出来. 基于客体分 子的易接近性以及酸位的稳定性, 选取分子篮 ZSM-5 中 Si12-O24(H)-Al12 位点来表示其酸性 中心. ${ }^{23}$ 在搭建模型中, 末端 $\mathrm{Si}$ 原子用 $\mathrm{H}$ 原子来饱 和, $\mathrm{H}$ 原子的取向与原晶体中被取代的氧原子取向 一致, 并且所有末端 $\mathrm{Si}-\mathrm{H}$ 键长设置为 $0.147 \mathrm{~nm}$.

\subsection{DFT 计算方法}

所有的理论计算都采用 Gaussian 09 软件包 ${ }^{24}$ 完 成. 随着模型选择的增大, 体系原子增多(例如 $128 \mathrm{~T}$ 模型, 结构式为 $\mathrm{Si}_{127} \mathrm{Al}_{1} \mathrm{O}_{219} \mathrm{H}_{75}$, 共 422 个原子), 对于 如此庞大的体系如果选择单纯的 DFT 方法因计算 量太大而在现有的计算水平下显然无法实现. 为了 提高计算效率, 在这里采用分子力学和量子力学结 合的 ONIOM (our own N-layer integrated MO and MM method) 方法, ${ }^{25,26}$ 该方法已成功应用于研究分 子篮的催化反应机理. ${ }^{27}$ 在计算中, 将体系分为两
层, 其中活性中心采用比较精确的量子力学方法(比 如 B3LYP、M06-2X 和 B97), 体系中其余的部分则用 较低水平的半经验(MNDO)方法加以处理. 这样可 以保证较好的计算精度, 同时也能减小计算量, 提 高计算效率. 在 30T-128T簇模型体系中, 高层为 $8 \mathrm{~T}$ 区域 $(\mathrm{SiO})_{3} \mathrm{Si}-\mathrm{OH}-\mathrm{Al}(\mathrm{OSi})_{3}$ 与吸附的吡定分子, 优 化分别采用 B3LYP、B971、B97D 和 M062X 方法, 基 组为 6-31G $(d, p)$; 剩余的部分为低层, 采用 $\mathrm{MNDO}$ 半经验方法. 在结构优化过程中, 只将分子篮的活 性中心部位 $\left(\mathrm{O}_{3} \mathrm{Si}-\mathrm{OH}-\mathrm{AlO}_{3}\right)$ 与吡定分子进行全优 化, 剩下的原子都被固定在原来的晶格位置, 这样 可以保持分子篮模型在结构优化的过程中不失去 其特有的晶体结构. 对于小的 8T ZSM-5 结构优化 采用同样的密度泛函方法以及基组进行计算, 在结 构优化过程中对 $\mathrm{O}_{3} \mathrm{Si}-\mathrm{OH}-\mathrm{AlO}_{3}$ 酸中心和比啶分 子进行优化, 而末端一 $\mathrm{SiH}_{3}$ 基团都固定在原来的晶 体位置. 单点能计算分别选用 B3LYP、B971、B97D 和 M06-2X 方法, 基组为 6-31G $(d, p)$. 而 B971-D 和 B3LYP-D 的单点能则是在 B971 和 B3LYP 方法得到 的单点能基础上加上相应的 $E_{\text {disp }}$ 校正.
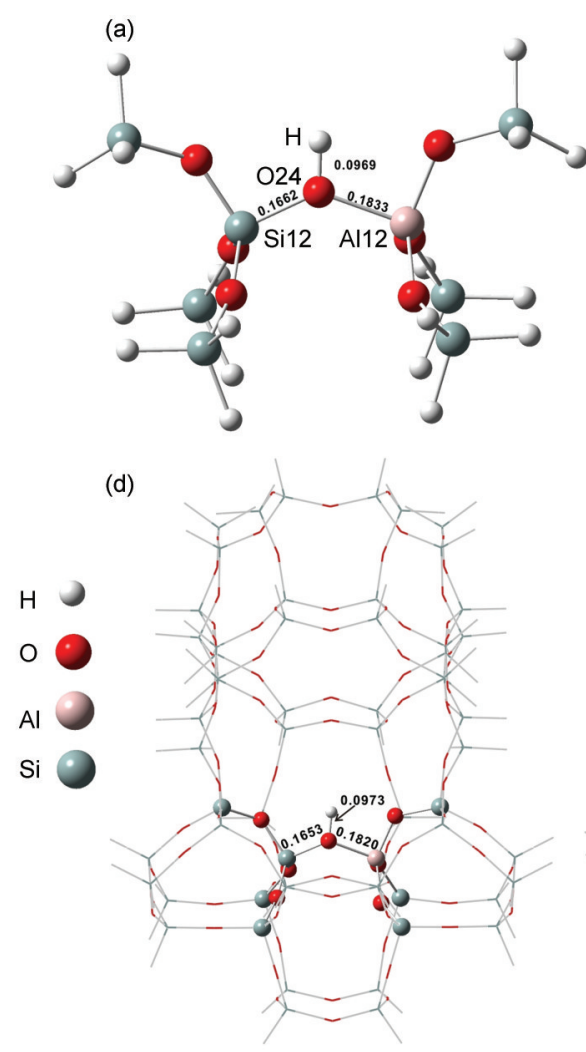

(b)

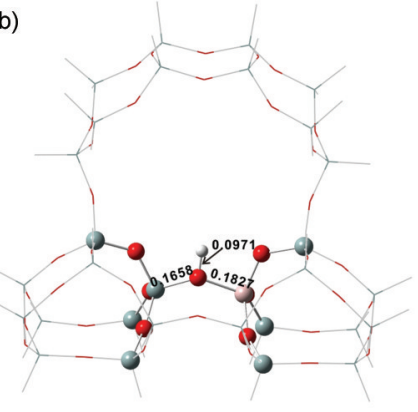

(e)

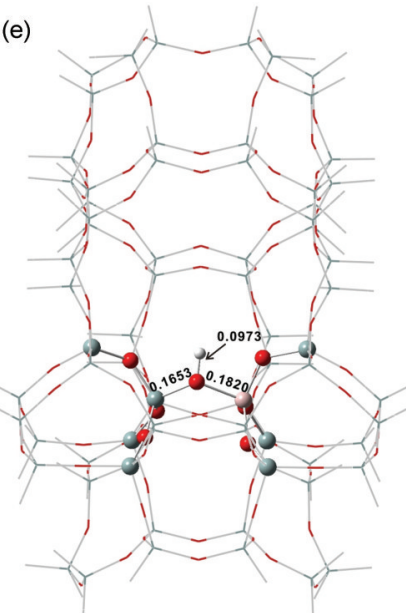

(c)

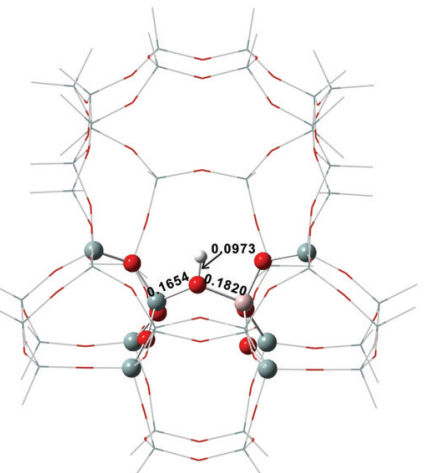

(f)

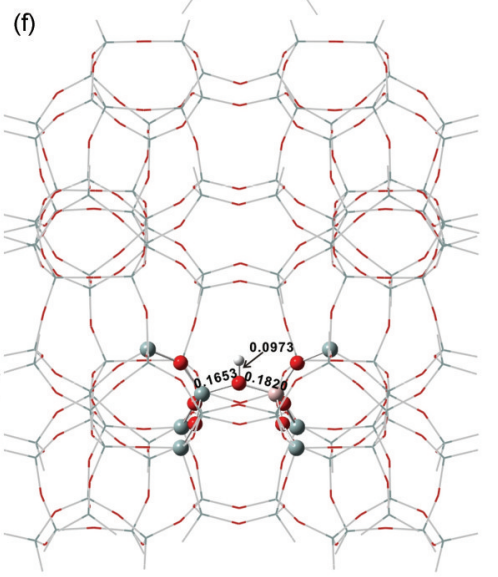

图 1 B3LYP/6-31G $(d, p)$ 水平下 8T-128T ZSM-5 模型优化得到的 Brönsted 酸位结构

Fig.1 Optimized structures of Brönsted acid sites with 8T-128T ZSM-5 models at B3LYP/6-31G $(d, p)$ level (a) $8 \mathrm{~T}$, (b) $30 \mathrm{~T}$, (c) 46T, (d) 60T, (e) $72 \mathrm{~T}$ (f) 128T; distances in $\mathrm{nm}$ 


\section{3 色散相互作用 $\left(E_{\text {disp }}\right)$ 的计算}

Grimme ${ }^{17}$ 和 Sumpter ${ }^{28}$ 提出长程相互作用的色 散能贡献可以用公式(1)表示:

$$
E_{\mathrm{disp}}=-S_{6} \sum_{i=1}^{N_{\mathrm{at}}-1} \sum_{j=i+1}^{N_{\mathrm{at}}} \frac{\sqrt{C_{6}^{i} C_{6}^{j}}}{R_{i j}^{6}} f_{\mathrm{damp}}\left(R_{i j}\right)
$$

在这里 $N_{\text {at }}$ 为体系中的原子数, $S_{6}$ 为校正系数, 通常 不同的泛函对应不同的校正系数, 比如 PBE 的校正 系数为 $0.75, \mathrm{~B} 3 \mathrm{LYP}$ 为 $1.05, \mathrm{~B} 97 \mathrm{D}$ 为 $1.25 ;{ }^{17} C_{6}$ 为原 子的色散系数, 其大小与原子的电离电势和静态偶 极化率相关; $f_{\text {damp }}\left(R_{i j}\right)$ 是与原子间距离 $R_{i j}$ 相关的阻尼 函数, 其表达式可以用公式(2)表示:

$$
f_{\text {damp }}\left(R_{i j}\right)=\left\{1+\exp \left[-\alpha\left(R_{i j} / R^{0}-1\right)\right]\right\}^{-1}
$$

其中 $R^{0}$ 为原子的半径, $i$ 和 $j$ 分别为体系中 $i$ 和 $j$ 两个 原子的原子序号, $R_{i j}$ 为 $i$ 和 $j$ 两原子间的距离. 表 1 给 出了 $\mathrm{H} 、 \mathrm{C} 、 \mathrm{O} 、 \mathrm{~N} 、 \mathrm{Al}$ 和 $\mathrm{Si}$ 原子的色散系数 $C_{6}$ 和原子 半径 $R^{0}$ 等有关参数. ${ }^{25}$ 基于公式(1)和公式(2), 色散相 互作用 $\left(E_{\mathrm{disp}}\right)$ 的计算由本课题组编写的 $\mathrm{C}$ 程序完成.

\section{3 结果与讨论}

表 2 不同计算方法下 8T-128T ZSM-5 模型去质子化能(DPE)、H 的电荷 $\left(Q_{\mathrm{H}}\right)$ 和主要结构参数

Table 2 Deprotonation energy (DPE), Mulliken charge for $\mathrm{H}\left(Q_{\mathrm{H}}\right)$, and main geometry parameters of 8T-128T ZSM-5

\begin{tabular}{|c|c|c|c|c|c|c|c|}
\hline \multirow{2}{*}{ Method } & \multirow{2}{*}{$\begin{array}{c}\text { ZSM-5 } \\
\text { cluster model }\end{array}$} & \multirow{2}{*}{$\mathrm{DPE} /\left(\mathrm{kJ} \cdot \mathrm{mol}^{-1}\right)$} & \multirow{2}{*}{$Q_{\mathrm{H}} /|e|$} & \multicolumn{4}{|c|}{ Geometry parameters } \\
\hline & & & & $r_{\mathrm{O}-\mathrm{H}} / \mathrm{nm}$ & $r_{\mathrm{Al}-\mathrm{O}} / \mathrm{nm}$ & $r_{\mathrm{Si}-\mathrm{o}} / \mathrm{nm}$ & $<\mathrm{AlOSi}>/\left(^{\circ}\right)$ \\
\hline \multirow[t]{6}{*}{ B3LYP } & $8 \mathrm{~T}$ & 1257.5 & 0.388 & 0.0969 & 0.1833 & 0.1662 & 130.38 \\
\hline & $30 \mathrm{~T}$ & 1256.2 & 0.397 & 0.0971 & 0.1827 & 0.1658 & 128.19 \\
\hline & $46 \mathrm{~T}$ & 1235.7 & 0.401 & 0.0973 & 0.1820 & 0.1654 & 119.04 \\
\hline & $60 \mathrm{~T}$ & 1235.6 & 0.401 & 0.0973 & 0.1820 & 0.1653 & 118.92 \\
\hline & $72 \mathrm{~T}$ & 1235.6 & 0.401 & 0.0973 & 0.1820 & 0.1653 & 118.91 \\
\hline & $128 \mathrm{~T}$ & 1235.6 & 0.401 & 0.0973 & 0.1820 & 0.1653 & 118.90 \\
\hline \multirow[t]{6}{*}{ B971 } & $8 \mathrm{~T}$ & 1289.5 & 0.393 & 0.0968 & 0.1838 & 0.1664 & 130.26 \\
\hline & $30 \mathrm{~T}$ & 1259.0 & 0.403 & 0.0973 & 0.1830 & 0.1659 & 127.80 \\
\hline & $46 \mathrm{~T}$ & 1236.2 & 0.406 & 0.0973 & 0.1827 & 0.1657 & 118.35 \\
\hline & $60 \mathrm{~T}$ & 1236.0 & 0.406 & 0.0973 & 0.1827 & 0.1657 & 118.27 \\
\hline & $72 \mathrm{~T}$ & 1236.0 & 0.406 & 0.0973 & 0.1827 & 0.1656 & 118.26 \\
\hline & $128 \mathrm{~T}$ & 1236.0 & 0.406 & 0.0973 & 0.1827 & 0.1656 & 118.25 \\
\hline \multirow[t]{6}{*}{ B97D } & $8 \mathrm{~T}$ & 1295.2 & 0.385 & 0.0972 & 0.1853 & 0.1672 & 128.01 \\
\hline & $30 \mathrm{~T}$ & 1261.8 & 0.395 & 0.0977 & 0.1850 & 0.1669 & 124.49 \\
\hline & $46 \mathrm{~T}$ & 1233.5 & 0.398 & 0.0977 & 0.1846 & 0.1667 & 115.94 \\
\hline & $60 \mathrm{~T}$ & 1233.8 & 0.398 & 0.0977 & 0.1846 & 0.1666 & 115.82 \\
\hline & $72 \mathrm{~T}$ & 1233.8 & 0.398 & 0.0977 & 0.1847 & 0.1666 & 115.80 \\
\hline & $128 \mathrm{~T}$ & 1233.8 & 0.398 & 0.0977 & 0.1847 & 0.1666 & 115.80 \\
\hline \multirow[t]{6}{*}{ M06-2X } & $8 \mathrm{~T}$ & 1286.1 & 0.412 & 0.0969 & 0.1833 & 0.1662 & 130.38 \\
\hline & $30 \mathrm{~T}$ & 1255.5 & 0.423 & 0.0973 & 0.1821 & 0.1654 & 128.29 \\
\hline & $46 \mathrm{~T}$ & 1236.8 & 0.426 & 0.0973 & 0.1813 & 0.1649 & 119.29 \\
\hline & $60 \mathrm{~T}$ & 1236.8 & 0.426 & 0.0973 & 0.1813 & 0.1648 & 119.27 \\
\hline & $72 \mathrm{~T}$ & 1236.8 & 0.426 & 0.0973 & 0.1813 & 0.1648 & 119.27 \\
\hline & $128 \mathrm{~T}$ & 1236.8 & 0.426 & 0.0973 & 0.1813 & 0.1648 & 119.27 \\
\hline
\end{tabular}
models calculated with different methods $\left(C_{6}\right)$ 和原子半径 $\left(R^{0}\right)^{25}$

Table 1 Dispersion coefficient $\left(C_{6}\right)$ and radii $\left(R^{0}\right)$ for elements $\mathrm{H}, \mathrm{C}, \mathrm{N}, \mathrm{O}, \mathrm{Al}$, and $\mathrm{Si}$ in the calculations of dispersion energy ${ }^{25}$

\begin{tabular}{ccc}
\hline Element & $C_{6} /\left(\mathrm{J} \cdot \mathrm{nm}^{6} \cdot \mathrm{mol}^{-1}\right)$ & $R^{0} / \mathrm{nm}$ \\
\hline $\mathrm{H}$ & 0.14 & 0.0000 \\
$\mathrm{C}$ & 1.75 & 0.1485 \\
$\mathrm{~N}$ & 1.23 & 0.1452 \\
$\mathrm{O}$ & 0.70 & 0.1397 \\
$\mathrm{Al}$ & 10.79 & 0.1364 \\
$\mathrm{Si}$ & 9.23 & 0.1639 \\
\hline
\end{tabular}

\section{1 分子篮酸性特征的理论研究}

分子篮的酸性特征将直接影响到吸附的有机物 种的结构和催化反应活性. van Santen ${ }^{29}$ 和 Blowers ${ }^{30}$ 等研究了固体酸酸强度对烷烃活化反应(如氢交换、 裂解、脱氢等)活化能垒的影响, 发现催化活性随着 酸强度的增大而显著增强. 在前期的研究工作中, 我们 $6,31,32$ 发现固体酸的酸强度也将影响到探针分子 的吸附和谱学参数, 固体酸的酸性越强, 酸性质子 向吡啶、三甲基膦氧等碱性探针分子转移的程度就 越显著, 同时探针分子的化学位移也表现出规律性
表 1 色散能计算中 $\mathrm{H} 、 \mathrm{C} 、 \mathrm{~N} 、 O 、 A I$ 和 $\mathrm{Si}$ 原子的色散系数 
的变化. 为了讨论固体酸模型大小对酸性中心的结 构和酸强度的影响, 基于 $8 \mathrm{~T} 、 30 \mathrm{~T} 、 46 \mathrm{~T} 、 60 \mathrm{~T} 、 72 \mathrm{~T}$ 和 $128 \mathrm{~T}$ 模型的计算得到的 Brönsted 酸中心的结构参 数和质子的 Mulliken 电荷 $\left(Q_{\mathrm{H}}\right)$ 列于表 2 中. 可以发现 不论采用哪种泛函方法, 随着模型的增大结构参数 呈现出相同的变化规律. 以 B3LYP 方法为例, 随着 模型的增大, $30 \mathrm{~T} 、 46 \mathrm{~T}$ 模型的 $\mathrm{Si}-\mathrm{O}$ 键长分别从 $8 \mathrm{~T}$ 模型的 $0.1662 \mathrm{~nm}$ 减小为 0.1658 和 $0.1654 \mathrm{~nm}$, 而 $\mathrm{Al}-\mathrm{O}$ 键长则在 $0.1833 \mathrm{~nm}$ 的基础上分别减小了 0.0006 和 $0.0013 \mathrm{~nm}$, 说明模型的增大 (从 $8 \mathrm{~T}$ 到 $46 \mathrm{~T}$ ) 对酸位的局域骨架结构有一定的影响; 而 46T 增大 到 $128 \mathrm{~T}$ 模型的结构参数变化则不明显, 说明对于 46T 以上模型, 其结构参数已经达到收玫, 模型的进 一步增大对骨架结构的影响很小. 同样, 对于羟基 $\mathrm{O}-\mathrm{H}$ 键长, $8 \mathrm{~T}$ 模型为 $0.0969 \mathrm{~nm}, 30 \mathrm{~T}$ 和 $46 \mathrm{~T}$ 分别为 0.0971 和 $0.0973 \mathrm{~nm}, 46 \mathrm{~T}$ 以上模型 $\mathrm{O}-\mathrm{H}$ 键长维持 在 $0.0973 \mathrm{~nm}$ 不变; $Q(\mathrm{H})$ 即 $\mathrm{H}$ 的电荷从 $0.388 \mid e$ 逐渐 增加到 $0.401|e|$ 后保持不变, 同样在 $46 \mathrm{~T}$ 呈现收玫的 趋势.

分子篮的催化反应活性是与其酸性特征密切
相关的, 通过 $r_{\mathrm{O}-\mathrm{H}}$ 和 Brönsted 质子 $Q_{\mathrm{H}}$ 的变化可以定 性地观察酸强度的变化趋势. 从表 2 给出的 $r_{\mathrm{O}-\mathrm{H}}$ 和 $Q_{\mathrm{H}}$ 的数据来看, 分子笁的酸强度随着选用模型的增 加而缓慢增强. 除此之外, 去质子化能(DPE) 是一种 有效表征酸强度大小的定量方法. ${ }^{33} \mathrm{DPE}$ 定义为酸 位脱去一个质子所要吸收的能量 $\left(\mathrm{AH} \rightarrow \mathrm{H}^{+}+\mathrm{A}^{-}\right)$, 在 数值上表现为酸位 $(\mathrm{AH})$ 的能量与失去质子后形成 共轭碱 $\left(\mathrm{A}^{-}\right)$的能量差值: $\mathrm{DPE}=E\left(\mathrm{~A}^{-}\right)-E(\mathrm{AH})$. 较小的 DPE 代表较强的酸性, 在酸催化反应中质子转移就 较容易. 从表 2 中发现 $8 \mathrm{~T} 、 30 \mathrm{~T} 、 46 \mathrm{~T} 、 60 \mathrm{~T} 、 72 \mathrm{~T}$ 和 $128 \mathrm{~T}$ 的 DPE 计算值 (由 B3LYP 方法计算得到) 分别是 $1257.5 、 1256.2 、 1235.7 、 1235.6 、 1235.6$ 和 $1235.6 \mathrm{~kJ}$. $\mathrm{mol}^{-1}$, 呈先降低后收玫的趋势; 不同的固体酸模型 的 DPE 值与 ZSM-5 分子篮的实验值范围 1217.8$1255.5 \mathrm{~kJ} \cdot \mathrm{mol}^{-134}$ 接近. 如表 2 所示, 这种变化趋势 在使用其他方法计算的结果中也有类似的体现. 这 说明分子篮的酸强度主要取决于酸性中心的局域 结构, 而孔道结构的影响不大.

\section{2 不同计算模型研究吡啶在分子䇻孔道中的吸附} 分子篮孔道和被吸附分子之间的主客体相互

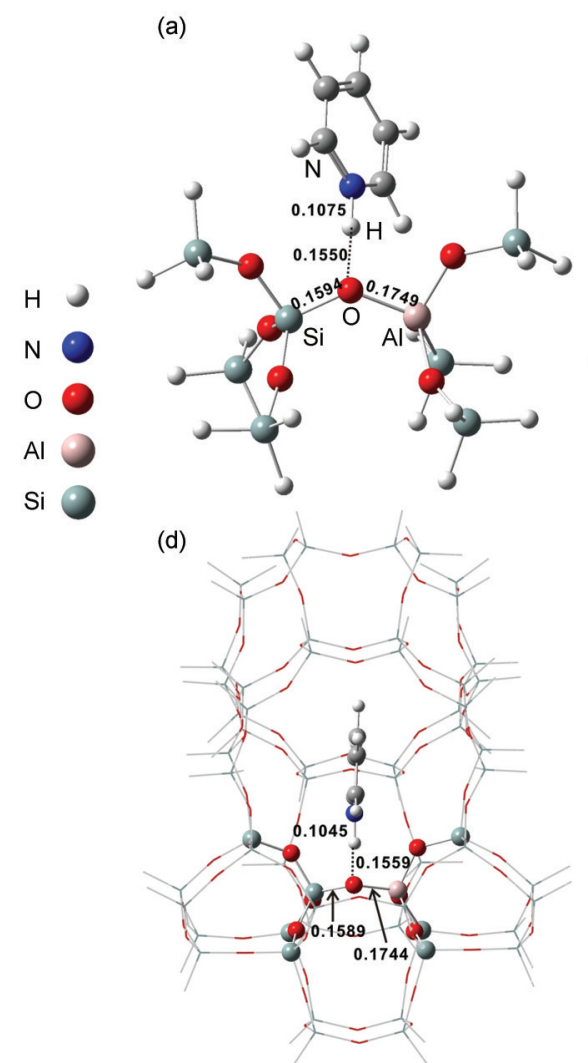

(b)

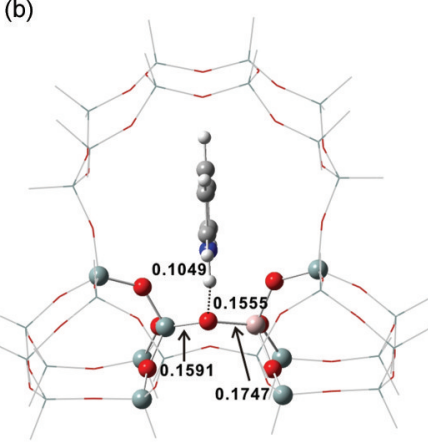

(e)

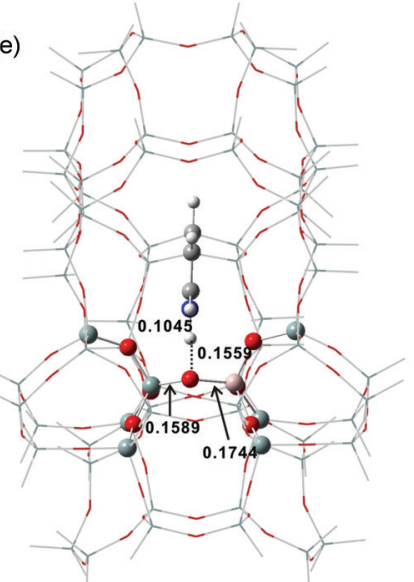

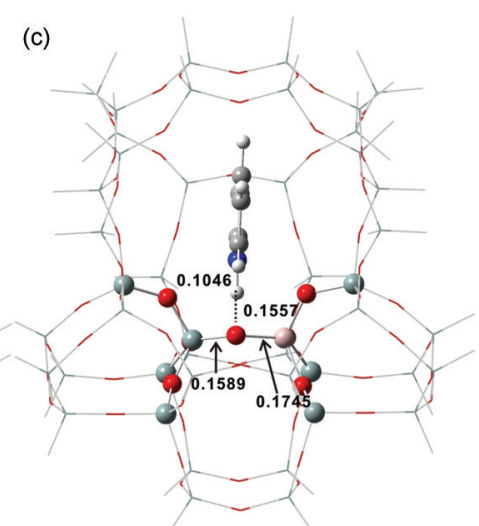

(f)

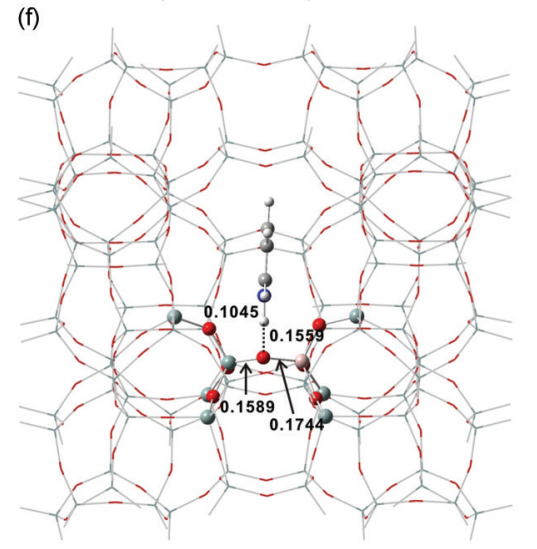

图 2 B3LYP/6-31G $(d, p)$ 方法下 8T-128T ZSM-5 模型吸附吡啶后优化得到的结构

Fig.2 B3LYP/6-31G $(d, p)$ optimized structures of pyridine adsorption complexes on 8T-128T ZSM-5 models (a) $8 \mathrm{~T}$, (b) $30 \mathrm{~T}$, (c) $46 \mathrm{~T}$, (d) $60 \mathrm{~T}$, (e) $72 \mathrm{~T}$, (f) $128 \mathrm{~T}$; distance in $\mathrm{nm}$ 
表 3 不同计算方法下 8T-128T ZSM-5 模型吸附吡啶后 $\mathrm{H}$ 的电荷和主要结构参数

Table 3 Mulliken charge for $\mathrm{H}$ and main geometry parameters of pyridine adsorption complexes on 8T-128T ZSM-5 models calculated with different methods

\begin{tabular}{|c|c|c|c|c|c|c|c|}
\hline \multirow{2}{*}{ Method } & \multirow{2}{*}{$\begin{array}{c}\text { ZSM-5 } \\
\text { cluster model }\end{array}$} & \multirow{2}{*}{$Q_{\mathrm{H}} /|e|$} & \multicolumn{5}{|c|}{ Geometry parameters } \\
\hline & & & $r_{\mathrm{N}-\mathrm{H}} / \mathrm{nm}$ & $r_{\mathrm{O}-\mathrm{H}} / \mathrm{nm}$ & $r_{\mathrm{Al}-\mathrm{O}} / \mathrm{nm}$ & $r_{\mathrm{Si}-\mathrm{O}} / \mathrm{nm}$ & $<\mathrm{AlOSi}>/\left(^{\circ}\right)$ \\
\hline \multirow[t]{6}{*}{ B3LYP } & $8 \mathrm{~T}$ & 0.420 & 0.1075 & 0.1550 & 0.1749 & 0.1594 & 129.34 \\
\hline & $30 \mathrm{~T}$ & 0.405 & 0.1049 & 0.1555 & 0.1747 & 0.1591 & 127.37 \\
\hline & $46 \mathrm{~T}$ & 0.401 & 0.1046 & 0.1557 & 0.1745 & 0.1589 & 121.53 \\
\hline & $60 \mathrm{~T}$ & 0.400 & 0.1045 & 0.1559 & 0.1744 & 0.1589 & 121.50 \\
\hline & $72 \mathrm{~T}$ & 0.400 & 0.1045 & 0.1559 & 0.1744 & 0.1589 & 121.50 \\
\hline & $128 \mathrm{~T}$ & 0.400 & 0.1045 & 0.1559 & 0.1744 & 0.1589 & 121.50 \\
\hline \multirow[t]{6}{*}{ B971 } & $8 \mathrm{~T}$ & 0.436 & 0.1077 & 0.1552 & 0.1753 & 0.1595 & 129.01 \\
\hline & $30 \mathrm{~T}$ & 0.419 & 0.1049 & 0.1561 & 0.1752 & 0.1593 & 125.45 \\
\hline & $46 \mathrm{~T}$ & 0.415 & 0.1047 & 0.1565 & 0.1751 & 0.1592 & 120.69 \\
\hline & $60 \mathrm{~T}$ & 0.412 & 0.1045 & 0.1567 & 0.1751 & 0.1592 & 120.67 \\
\hline & $72 \mathrm{~T}$ & 0.412 & 0.1045 & 0.1567 & 0.1751 & 0.1592 & 120.67 \\
\hline & $128 \mathrm{~T}$ & 0.412 & 0.1045 & 0.1567 & 0.1751 & 0.1592 & 120.67 \\
\hline \multirow[t]{6}{*}{ B97D } & $8 \mathrm{~T}$ & 0.419 & 0.1083 & 0.1543 & 0.1762 & 0.1602 & 126.93 \\
\hline & $30 \mathrm{~T}$ & 0.405 & 0.1058 & 0.1559 & 0.1761 & 0.1600 & 125.52 \\
\hline & $46 \mathrm{~T}$ & 0.400 & 0.1050 & 0.1560 & 0.1760 & 0.1600 & 121.92 \\
\hline & $60 \mathrm{~T}$ & 0.395 & 0.1047 & 0.1563 & 0.1759 & 0.1600 & 121.84 \\
\hline & $72 \mathrm{~T}$ & 0.395 & 0.1047 & 0.1563 & 0.1758 & 0.1598 & 121.83 \\
\hline & $128 \mathrm{~T}$ & 0.395 & 0.1047 & 0.1563 & 0.1758 & 0.1598 & 121.83 \\
\hline \multirow[t]{6}{*}{ M06-2X } & $8 \mathrm{~T}$ & 0.455 & 0.1096 & 0.1467 & 0.1748 & 0.1593 & 130.54 \\
\hline & $30 \mathrm{~T}$ & 0.431 & 0.1053 & 0.1551 & 0.1744 & 0.1589 & 129.62 \\
\hline & $46 \mathrm{~T}$ & 0.423 & 0.1049 & 0.1552 & 0.1742 & 0.1588 & 121.83 \\
\hline & $60 \mathrm{~T}$ & 0.421 & 0.1047 & 0.1555 & 0.1741 & 0.1588 & 121.83 \\
\hline & $72 \mathrm{~T}$ & 0.421 & 0.1047 & 0.1555 & 0.1741 & 0.1588 & 121.83 \\
\hline & $128 \mathrm{~T}$ & 0.421 & 0.1047 & 0.1555 & 0.1741 & 0.1588 & 121.83 \\
\hline
\end{tabular}

作用将直接影响到吸附分子的构型以及其物理化 学性质. 被吸附分子与分子篮孔道之间主要是静电 吸引和范德华作用, 当被吸附分子逐渐被孔道包围 时, 这种稳定作用也逐渐增强, 即“孔道限域效应”。 在研究了不同大小的分子笁模型对其固有酸强度 的影响之后, 我们将进一步研究孔道效应对吡啶结 构的影响. 图 2 给出了 B3LYP 计算方法下 ZSM-5 不 同酸模型的吡啶吸附结构图. 比较图 1 和图 2 中分 子篮和吡啶的构型变化后可以发现, 在吡啶吸附前 后 $r_{\mathrm{O}-\mathrm{H}}$ 键长的变化是非常明显的. 以 $72 \mathrm{~T}$ 模型为例, 吡定吸附前, $\mathrm{O}-\mathrm{H}$ 键长为 $0.0973 \mathrm{~nm}$, 吸附吡啶后, $\mathrm{O}-\mathrm{H}$ 键长增大为 $0.1559 \mathrm{~nm}$, 而 $\mathrm{N}-\mathrm{H}$ 键长为 $0.1045 \mathrm{~nm}$, 这表明吡啶被质子化形成了吡啶离子. 相应的, 吡啶吸附前后 Brönsted 酸位上的 $\mathrm{O}$ 原子与 $\mathrm{Si}$ 和 $\mathrm{Al}$ 的距离也呈现一定的趋势: 对于 $8 \mathrm{~T}$ 模型, $\mathrm{O}-\mathrm{Al}$ 和 $\mathrm{O}-\mathrm{Si}$ 键长分别为 0.1833 和 $0.1662 \mathrm{~nm}$; 在 吡啶探针分子作用下, $\mathrm{O}-\mathrm{Al}$ 和 $\mathrm{O}-\mathrm{Si}$ 键长分别减 小为 0.1749 和 $0.1594 \mathrm{~nm}$; 对于 $72 \mathrm{~T}$ 模型, 吡啶吸附 前后 $\mathrm{O}-\mathrm{Al}$ 和 $\mathrm{O}-\mathrm{Si}$ 键长分别从 0.1820 和 0.1653 $\mathrm{nm}$ 减小为 0.1744 和 $0.1589 \mathrm{~nm}$. 表 3 给出了在不同
模型和方法下计算得到质子的 Mulliken 电荷 $\left(Q_{\mathrm{H}}\right)$ 和 结构参数. 研究结果表明不论采用哪种泛函方法, 随着模型的增大, $E_{\mathrm{ads}} 、 Q_{\mathrm{H}}$ 以及结构参数都呈现出相 同的变化规律. 以 B3LYP 方法为例,随着酸模型的增 大, $\mathrm{N}-\mathrm{H}$ 键长逐渐从 $0.1075 \mathrm{~nm}$ 减小到 $0.1045 \mathrm{~nm}$, 而后者与气态吡啶离子的 $\mathrm{N}-\mathrm{H}$ 键长 $0.1017 \mathrm{~nm}$ 是 比较接近的. 另外通过比较不同模型间的参数变化 发现, $8 \mathrm{~T}$ 和 $30 \mathrm{~T}$ 模型间的参数变化为 $\Delta r_{\mathrm{N}-\mathrm{H}}=0.0026$ $\mathrm{nm}, \Delta r_{\mathrm{O}-\mathrm{H}}=0.0005 \mathrm{~nm} ; 30 \mathrm{~T}$ 和 $46 \mathrm{~T}$ 之间变化很小 $\Delta r_{\mathrm{N}-\mathrm{H}}=0.0003 \mathrm{~nm}, \Delta r_{\mathrm{O}-\mathrm{H}}=0.0002 \mathrm{~nm}$, 而 $60 \mathrm{~T}$ 到 $128 \mathrm{~T}$ 之间则基本没有变化. 值得注意的是, 在 $8 \mathrm{~T}$ 模型中, $Q_{\mathrm{H}}$ 为 $0.420|e|$, 而在 $72 \mathrm{~T}$ 模型中 $Q_{\mathrm{H}}$ 减小为 $0.400|e|$. 这 说明随着酸模型的增大, 越来越多的正电荷被分散 到吡啶分子的共轭环中, 增大了吡啶离子的稳定 性. 这也进一步说明了孔道结构对吡啶吸附起到了 稳定的作用. 不同方法计算得到的吡啶吸附参数有 一定的差别, 说明不同的计算方法对吡啶在分子篮 表面吸附的构型是有影响的; 但从 $8 \mathrm{~T}$ 到 $128 \mathrm{~T}$ 模型 参数的变化趋势上看, 无论哪一种泛函方法, 其整 体变化趋势都是一致的. 
表 4 给出了吡啶在不同的酸模型表面上的吸附 能. 吸附能计算公式如下:

$$
\Delta E_{\text {ads }}=\left(E_{\text {zOH }}+E_{\text {pyridine }}\right)-E_{\text {pyridine-ZOH }}
$$

其中 $E_{\mathrm{pyridine}-\mathrm{ZOH}}$ 为复合物的能量, $E_{\mathrm{ZOH}}$ 和 $E_{\mathrm{pyridine}}$ 分别为 分子篮模型和吡啶分子的单点能. 在这里需要提出 的是, 计算得到的吸附能是体系在绝对零度的相互 作用能, 其值与实验得到的吸附热接近. ${ }^{35,36}$ 表 4 给出 的吡啶吸附能结果分析发现, 随着模型的增大, 吸 附能呈增大趋势并最后达到收敛. 对于 B3LYP 方 法, $8 \mathrm{~T}$ 模型的吸附能为 $45.4 \mathrm{~kJ} \cdot \mathrm{mol}^{-1}$, 它包含了酸位 的局域结构, 但没考虑孔道; 30T 和 $46 \mathrm{~T}$ 模型吸附能 分别为 85.4 和 $88.9 \mathrm{~kJ} \cdot \mathrm{mol}^{-1}$, 与 $8 \mathrm{~T}$ 模型相比吸附能 分别升高了 40 和 $43.5 \mathrm{~kJ} \cdot \mathrm{mol}^{-1}$, 这是由于 $30 \mathrm{~T}$ 和 $46 \mathrm{~T}$ 模型既包含了酸位的局域结构, 又包含了部分 孔道; 60T、72T 和 $128 \mathrm{~T}$ 模型的吸附能分别为 90.0 、 90.4 和 $90.6 \mathrm{~kJ} \cdot \mathrm{mol}^{-1}$, 模型之间能量只有 $0.6 \mathrm{~kJ}$. $\mathrm{mol}^{-1}$ 的变化, 说明孔道结构已经逐渐完整, 对于吡 啶的吸附影响变小, 能量达到收玫. 这说明对于复 杂的 ZSM-5 分子笁体系, 72T 模型可以完全将孔道 对吸附分子之间的相互作用都包括进来, 可以用来 反映 ZSM-5 分子篎真实的孔道结构. 对于其他方法 计算得到的吡啶吸附能(表 4)也同样表现出随着计 算模型的增大而增大, 在 $72 \mathrm{~T}$ 模型时能量达到收敛.

\section{3 不同泛函方法研究吡啶在分子篮孔道中的吸附}

基于优化得到的吡定分子吸附结构, 我们在表 4 中给出了不同模型和计算方法下吡啶在 ZSM-5 分 子篮表面的吸附能. 在前面的研究中我们已经确定 了 72T 模型可以用来反映分子篮 ZSM-5 的孔道结 构, 所以在这里以 $72 \mathrm{~T}$ 模型为例比较不同计算方法 得到的吸附能. 在 B3LYP/6-31G $(d, p)$ 水平下计算得 到的吸附能为 $90.4 \mathrm{~kJ} \cdot \mathrm{mol}^{-1}$, 而实验值为 $200 \mathrm{~kJ}$. $\mathrm{mol}^{-1}{ }^{37}$ 众所周知对于质子化的吡啶离子与分子篎

\section{表 4 不同计算方法下得到的 8T-128T ZSM-5 模型吡啶} 吸附能 $\left(\boldsymbol{E}_{\text {ads }}\right)$

Table 4 Pyridine adsorption energies $\left(E_{\text {ads }}\right)$ on 8T-128T ZSM-5 models calculated with different methods

\begin{tabular}{rcrrcrc}
\hline $\begin{array}{c}\text { ZSM-5 } \\
\text { cluster } \\
\text { model }\end{array}$ & \multicolumn{6}{c}{$E_{\text {ads }} /\left(\mathrm{kJ} \cdot \mathrm{mol}^{-1}\right)$} \\
\cline { 2 - 7 } & B3LYP & B971 & M06-2X & B3LYP-D & B971-D & B97D \\
\hline $8 \mathrm{~T}$ & 45.4 & 67.5 & 114.0 & 71.3 & 79.4 & 132.4 \\
$30 \mathrm{~T}$ & 85.4 & 104.8 & 137.7 & 113.9 & 114.0 & 186.9 \\
$46 \mathrm{~T}$ & 88.9 & 109.2 & 143.3 & 121.5 & 121.7 & 200.0 \\
$60 \mathrm{~T}$ & 90.0 & 110.2 & 143.9 & 122.3 & 122.4 & 202.0 \\
$72 \mathrm{~T}$ & 90.4 & 110.4 & 144.3 & 122.4 & 122.5 & 204.7 \\
$128 \mathrm{~T}$ & 90.6 & 110.5 & 144.6 & 122.5 & 122.6 & 204.8 \\
\hline
\end{tabular}

孔道之间的相互作用, 除了电子相关效应外, 静电 吸引和范德华作用也起到稳定吡啶离子的作用. B3LYP 方法仅仅能对电子相关效应作出较好的描 述, 而不能够很好地描述范德华作用力, 因此计算 得到的吸附能与实验值误差很大. $\mathrm{B} 971 / 6-31 \mathrm{G}(d, p)$ 水平下计算得到的吸附能为 $110.4 \mathrm{~kJ} \cdot \mathrm{mol}^{-1}$, 与 B3LYP 方法相比计算精度稍有提高, 这是因为 B97 方法在电子相关方面进行了一定的修正, 对于研究 短程作用为主的体系有较高的计算精度, 但由于对 范德华作用力的描述依旧不是很全面, 所以计算得 到的吸附能与实验值仍有很大的误差. M06-2X方 法考虑了弱相互作用力, 在计算范德华作用方面性 能比 B3LYP 有所改善, 计算得到的吸附能量为 $144.3 \mathrm{~kJ} \cdot \mathrm{mol}^{-1}$, 与 B3LYP 相比计算精确明显提高. ${ }^{19}$ 但对于主客体间普遍存在的长程色散作用, M06-2X 未能体现出色散作用对吸附能量的影响, 对于分子 间的色散作用描述不是很充分, 计算结果与实验值 仍有约 30\%的误差. B3LYP-D 和 B971-D 分别是在 B3LYP 和 B971 优化后对色散作用进行校正, 因此 得到的能量比 B3LYP 和 B971 方法计算的结果进一 步提高, 分别为 122.4 和 $122.5 \mathrm{~kJ} \cdot \mathrm{mol}^{-1}$, 但这两种校 正方法的最大问题在于仅仅在单点能计算部分增 加了一项色散校正, 并没有将弱相互作用的影响引 入结构优化过程中, 虽然相对于纯的密度泛函计算 得到的能量有所改进, 但是与实验值相差仍然约 80 $\mathrm{kJ} \cdot \mathrm{mol}^{-1}$. B97D 方法是在 B97 的基础上, 每一步结 构优化的过程中都进行色散校正, 因此可以预期该 种方法得到的结构和能量精度将会有很大的提高. 从表 3 给出的不同方法下吡啶吸附的结构参数可以 发现, B97D 方法计算得到的 $\mathrm{N}-\mathrm{H}$ 和 $\mathrm{O}-\mathrm{H}$ 键长分 别为 0.1047 和 $0.1563 \mathrm{~nm}$, 比 B971 方法计算得到的 $\mathrm{N}-\mathrm{H}$ 键长增加了 $0.0002 \mathrm{~nm}, \mathrm{O}-\mathrm{H}$ 键长减小了 $0.0004 \mathrm{~nm}$; 基于 B97D 水平下得到的结构参数, 我们 进一步计算得到其吸附能为 $204.7 \mathrm{~kJ} \cdot \mathrm{mol}^{-1}$, 与实验 值 $200 \mathrm{~kJ} \cdot \mathrm{mol}^{-1}$ 吻合得较好, 这也进一步验证了 B97D 方法在计算色散作用力方面有较大的优势. ${ }^{38}$ 图 3 给出了不同团簇模型和不同方法下吡啶的吸附 能以及实验值, 从图中可以更直观地看出 B97D 方 法与实验值吻合得最好.

\section{4 分子節体系理论计算模型和方法的选择}

分子篮催化剂的优越性主要体现在其具有一 定的酸性和独特的孔道特征, 因此在选择计算模型 的时候需要充分考虑这两个因素. 通过系统地研究 


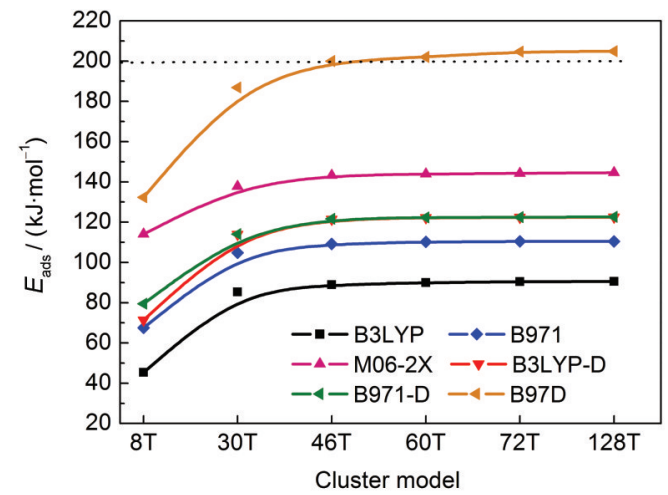

图 3 六种计算方法下 8T-128T ZSM-5 模型吡啶吸附能

Fig.3 Adsorption energies of pyridine on 8T-128T ZSM-5 models calculated with six different methods

The experimental value $\left(200 \mathrm{~kJ} \cdot \mathrm{mol}^{-1}\right)^{37}$ is shown by the dotted line.

发现分子篎的酸强度主要取决于酸性中心的局域 结构, 孔道结构对分子篮的固有酸强度影响不大. 从图 2 给出的吡啶吸附结构图和表 4 给出的吡啶吸 附能结果可以发现, 随着模型选择的增大, 孔道结 构逐渐完整, “孔道限域效应”越来越明显, 吸附能 呈增大趋势并在 60T-128T 达到收敛. 另外需要注 意的是, 随着模型的增大, 体系原子增多, 计算量也 相应增大. 基于以上因素考虑, 我们选用 $72 \mathrm{~T}$ 模型来 进行分子篮体系的理论计算, 因为 $72 \mathrm{~T}$ 模型既能表 现出 ZSM-5 的酸性特征, 又具备完整的孔道结构, 可以充分地反映 ZSM-5 的催化性能; 另外 72T 模型 原子数量适中, 计算量比 $128 \mathrm{~T}$ 小, 计算精度比小模 型高, 因此选用 $72 \mathrm{~T}$ 模型来代表 ZSM-5 分子篮的孔 道结构应用于一些理论计算可以得到很好的结果.

分子篮体系中的主客体间相互作用非常复杂, 除了电子相关效应外, 静电吸引、范德华作用以及 色散作用等长程相互作用对于有机物种在分子篮 孔道中的吸附都有不同程度的影响. 因此选择合适 的计算方法用于准确描述有机物种在分子篮孔道 中的吸附结构和能量, 对于解释实验现象、揭示反 应机理是非常重要的. 通过研究不同泛函方法计算 吡啶在分子篮孔道中吸附的结果发现, B97D 方法 可以很好地处理范德华作用等弱相互作用, 在计算 色散作用力方面也有较大的优势, 是一种能够准确 预测有机分子在分子篮孔道中结果参数和吸附能 量的准确方法.

\section{4 结 论}

通过量子化学计算的方法系统考察了计算中
分子篮模型和泛函方法对分子笁固有酸强度与吡 啶吸附结构和能量的影响. 结果表明, 分子篮的酸 强度主要取决于酸性中心的局域结构. 而在研究反 应物在分子篮限域孔道中吸附和转化过程中, 为了 考察吸附分子和分子篮孔道之间的主客体相互作 用, 有必要采用大的模型将分子篮完整的孔道结构 包括进来. 由于经典的 B 3 LYP 泛函方法未能将色散 作用力考虑进来, 采用该方法计算的吸附能量明显 低于实验值. 为了获得精确的结构和能量数据, B97D泛函方法在结构优化的每一步都作出色散作 用力的校正, 计算结果与实验值能够很好的吻合.

\section{References}

(1) Weitkamp, J.; Traa, Y. Catal. Today 1999, 49, 193.

(2) Slagtern, A.; Dahl, I. M.; Jens, K. J.; Myrstad, T. App. Catal. AGen. 2010, 375, 213.

(3) Luzgin, M. V.; Parmon, V. N. Angew. Chem. Int. Edit. 2008, 47, 4559.

(4) Yu, Z. W.; Zheng, A. M.; Wang, Q.; Huang, S. J.; Deng, F.; Liu, S. B. Chin. J. Magn. Reson. 2010, 27, 485. [喻志武, 郑安民, 王 强, 黃信炅, 邓 风, 刘尚斌. 波谱学杂志, 2010, 27, 485.]

(5) Zheng, A. M.; Huang, S. J.; Deng, F.; Liu, S. B. Phys. Chem. Chem. Phys. 2011, 13, 14889.

(6) Zheng, A. M.; Zhang, H. L.; Chen, L.; Yue, Y.; Ye, C. H.; Deng, F. J. Phys. Chem. B 2007, 111, 3085.

(7) Coma, A. Chem. Rev. 1995, 95, 559.

(8) Yuan, S. P.; Wang, J. G.; Li, Y. W.; Peng, S. Y. Acta PhysicoChimica Sinica 2001, 17,811. [袁淑萍, 王建国, 李永旺, 彭少逸. 物理化学学报, 2001, 17,811.]

(9) Yang, J.; Sun, Y. X.; Zhao, L. F.; Sun, H. Acta Physico-Chimica Sinica 2011, 27, 1823. [杨 静, 孙迎新, 赵立峰, 孙 淮. 物理化学学报, 2011, 27, 1823.]

(10) Yang, G.; Wang, Y.; Zhou, D. H.; Zhuang, J. Q.; Liu, X. C.; Han, X. W.; Bao, X. H. J. Chem. Phys. 2003, 119, 9765.

(11) Zhou, D. H.; Ma, D.; Liu, X. C.; Bao, X. H. J. Chem. Phys. 2001, 114, 9125.

(12) Hohenberg, P.; Kohn, W. Phys. Rev. 1964, 136, 864.

(13) Kohn, W.; Becke, A. D.; Parr, R. G. J. Phys. Chem. 1996, 100, 12974.

(14) Kristyan, S.; Pulay, P. Chem. Phys. Lett. 1994, 229, 175.

(15) Schmider, H. L.; Becke, A. D. J. Chem. Phys. 1998, 108, 9624.

(16) Zhao, Y.; Schultz, N. E.; Truhlar, D. G. J. Chem. Theory Comput. 2006, 2, 364.

(17) Grimme, S. J. Comput. Chem. 2004, 25, 1463.

(18) Grimme, S. J. Comput. Chem. 2006, 27, 1787.

(19) Zhao, Y.; Truhlar, D. G. J. Phys. Chem. C 2008, 112, 6860.

(20) Pidko, E. A.; Hensen, E. J. M.; van Santen, R. A. J. Phys. Chem. 
C 2008, 112, 19604.

(21) Boronat, M.; Martinez, C.; Corma, A. Phys. Chem. Chem. Phys. 2011, 13, 2603.

(22) Boekfa, B.; Choomwattana, S.; Khongpracha, P.; Limtrakul, J. Langmuir 2009, 22, 12990.

(23) Vankoningsveld, H.; Van Bekkum, H.; Jansen, J. C. Acta Crystallogr. B 1987, 43, 127.

(24) Frisch, M. J.; Trucks, G. W.; Schlegel, H. B.; et al. Gaussian 09, B.01; Gaussian Inc.: Wallingford, CT, 2009.

(25) Maseras, F.; Morokuma, K. J. Comput. Chem. 1995, 16, 1170.

(26) Humbel, S.; Siebe, R. S.; Morokuma, K. J. Chem. Phys. 1996, 105, 1959.

(27) Lesthaeghe, D.; Speybroeck, V. V.; Marin, G. B.; Waroquier, M. Chem. Phys. Lett. 2006, 417, 309.

(28) Sumpter, B. G. J. Chem. Theory Comput. 2010, 6, 727.

(29) Rigby, A. M.; Kramer, G. J.; van Santen, R. A. J. Catal. 1997, $170,1$.
(30) Zheng, X.; Blowers, P. J. Phys. Chem. A 2006, 110, 2455.

(31) Zheng, A. M.; Zhang, H. L.; Lu, X.; Liu, S. B.; Deng, F. J. Phys. Chem. B 2008, 112, 4496.

(32) Zheng, A. M.; Huang, S.; Chen, W.; Wu, P.; Zhang, H.; Lee, H.; Ménorval, L.; Deng, F.; Liu, S. B. J. Phys. Chem. A 2008, 112, 7337.

(33) Brand, H. V.; Curtiss, L. A.; Iton, L. E. J. Phys. Chem. 1993, 97, 12773.

(34) Datka, J.; Boczar, M.; Rymarowicz, P. J. Catal. 1988, 114, 368.

(35) Dunne, J. A.; Rao, M.; Sircar, S.; Corte, R. J.; Myers, A. L. Langmuir 1996, 12, 5896.

(36) Savitz, S.; Siperstein, F.; Rorte, R. J.; Myers, A. L. J. Phys. Chem. B 1998, 102, 6865.

(37) Lee, C.; Parrillo, D. J.; Gorte, R. J.; Farneth, W. E. J. Am. Chem. Soc. 1996, 118, 3262.

(38) Fuchs, A. H.; Adamo, C. J. Phys. Chem. Lett. 2010, 1, 763. 